\title{
Bildungs- und Schulmanagement-Studiengänge in Deutschland
}

\section{Zusammenfassung}

In Deutschland gibt es keine bundesweit verbindliche Einstiegsqualifizierung für Schulleitungen. Wer sich dennoch auf eine Leitungsfunktion vorbereiten will, kann einen der Bildungsmanagement-Studiengänge belegen, die im deutschsprachigen Raum (Deutschland, Österreich, deutschsprachige Schweiz, Regionen in Luxemburg) seit der Jahrtausendwende in wachsendem Maße angeboten werden. Im folgenden Werkstattbericht wird den Fragen nachgegangen, wie die Angebotslandschaft der Bildungsmanagement-Masterstudiengänge in Deutschland sich in den letzten Jahren entwickelt hat und wie sie aktuell beschrieben werden kann. Zudem wird skizziert, welches Lernwissen die Curricula vorsehen und welche Unterschiede auszumachen sind. Der Artikel begrenzt sich auf die Entwicklungen in Deutschland.

Schlagwörter: Wissenskanon Schulmanagement, Modulhandbuch, Wissensformen

\section{Study Programs of Education Management and School Management in Germany}

\begin{abstract}
In Germany, there is no compulsory and nation-wide standardized qualification for school principals. If teachers still want to prepare for a management position, they can register for one of the study programs in education management that have been offered increasingly since the turn of the millennium in German-speaking countries (Germany, Austria, German-speaking Switzerland, regions in Luxembourg). The following report investigates the offer of master's programs in education management in Germany and describes its development in recent years. The report also indicates which knowledge the curricula offer and which differences can be identified. The article focuses on developments in Germany.
\end{abstract}

Keywords: school management knowledge, module handbook, forms of knowledge 
Bildungs- und Schulmanagement-Studiengänge in Deutschland |

\section{Das Forschungsprojekt LeBiS}

Im Rahmen des Forschungsprojektes „Lernwissen der Bildungsmanagement-Studiengänge“ (LeBiS) wird an der Pädagogischen Hochschule Ludwigsburg seit 2013 das Lernwissen von Bildungsmanagement-Studiengängen erhoben (vgl. Krüger, 2016a). Schulmanagement-Studiengänge werden als Untergruppe der BildungsmanagementStudiengänge mit untersucht. Der Begriff „Lernwissen“ steht im Projekt für jenes Wissen, das im Rahmen eines Studiengangs offiziell erlernt werden soll und zu diesem Zweck in den Modulhandbüchern der Studiengänge skizziert ist. Als offizieller Lehrinhalt des Bildungsmanagements hat diese Wissensform Einfluss auf individuelle und gesellschaftliche Erwartungsstrukturen gegenüber Bildungsmanager*innen und damit auch gegenüber Schulleitungen. Die Grenzen sind hier fließend, da viele Studiengänge ihr Angebot sowohl für Schulleitungen als auch für Zielgruppen aus anderen Bildungssektoren erbringen. Sollte es je $\mathrm{zu}$ einer verbindlichen und standardisierten Ausbildung für Schulleitungen kommen, so wird die Bedeutung des dann aktuell bereits etablierten „offiziellen Wissenskanons des Bildungs- und Schulmanagements“ als Referenzwert steigen. Das LeBiS-Projekt begrenzt sich auf Bildungsmanagement-Studiengänge, die auf Master-Niveau im deutschsprachigen Raum angeboten werden.

\section{Angebotslandschaft der Bildungsmanagement- Masterstudiengänge im deutschsprachigen Raum}

Ein Bildungsmanagement-Studiengang im Sinne des Projektes ist ein Studiengang, der

- den Begriff „Bildungsmanagement“ oder eine sektorenspezifische Ableitung dieses Begriffs (Schulmanagement, Weiterbildungsmanagement, Hochschulmanagement etc.) im Titel führt oder

- den Anspruch vertritt, (unter anderem) explizit für ein Leitungshandeln in Bildungseinrichtungen oder Bildungsabteilungen zu qualifizieren oder

- das Organisationslernen in den Mittelpunkt stellt (Krüger, 2016b, S. 306).

Bezogen auf das Referenzsemester Sommer 2019 sind im gesamten deutschsprachigen Raum 62 solcher Masterstudiengänge auszumachen. In Deutschland werden 45 Bildungsmanagement-Studiengänge angeboten, die sich zum Teil auf einzelne Bildungssektoren wie die frühkindliche Bildung, die Schule oder die betriebliche Bildung beziehen und zum Teil sektorenübergreifend angelegt sind. Vier Masterstudiengänge bereiten in Deutschland sektorspezifisch auf Schulleitungsaufgaben vor. Diese werden an der TU Kaiserlautern, den Universitäten in Kiel und Tübingen sowie an der $\mathrm{PH}$ Weingarten angeboten. Hinzu kommen zahlreiche Angebote, die sich auf die Schule und mindestens einen weiteren Bildungssektor beziehen und Übergänge zwischen den Bildungssektoren thematisieren. Beispielhaft können hier 
die Studiengänge „Bildungsmanagement“ (PH Ludwigsburg) sowie „Schul- und Bildungsmanagement“ (Univ. Potsdam) genannt werden. Um den Horizont der Masterstudiengänge, die unter anderem für eine Schulleitungsqualifizierung genutzt werden, vollständig zu erfassen, erscheint es allerdings sinnvoll, das gesamte Spektrum der sektorenübergreifenden Bildungsmanagement-Studiengänge in den Blick zu nehmen. Denn wie wir aus Interviews wissen (vgl. Krüger, 2016a), werden diese Studiengänge zumindest punktuell ebenfalls von (angehenden) Schulleitungen mit dem Ziel besucht, sich für ihre Leitungstätigkeit zu qualifizieren. Durch diese Erweiterung rücken auch Studiengänge wie „Erziehungswissenschaft - Innovation und Management im Bildungswesen“ (Univ. Erfurt), „Leitung - Bildung - Diversität“ (EH Berlin) oder „International Education Management“ (PH Ludwigsburg) als mögliche Schulleitungsqualifizierungen in den Fokus. Insgesamt 27 Masterstudiengänge können somit in Deutschland als schulspezifische oder sektorenübergreifende Bildungsmanagement-Studiengänge identifiziert werden.

Die Anzahl der Bildungsmanagement-Studiengänge ist in den letzten 20 Jahren kontinuierlich gestiegen. Zwischen den Jahren 2013 und 2019 wurde das Angebot in Deutschland von 37 auf 45 Studiengänge ausgebaut, was einem Wachstum von fast 22 Prozent entspricht. Im gesamten deutschsprachigen Raum stieg das Angebot um 17 Prozent. Allerdings darf dabei nicht übersehen werden, dass der Markt der zumeist weiterbildenden und damit in der Regel zahlungspflichtigen Studiengänge eine hohe Dynamik aufweist. Zwischen den beiden Erhebungszeitpunkten haben neun Bildungsmanagement-Studiengänge den Betrieb eingestellt, während 17 Angebote neu aufgebaut wurden. Es müssen daher zwei Effekte unterschieden werden.

1) Ein Zuwachs an Bildungsmanagement-Studiengängen ist zwischen 2013 und 2019 maßgeblich im Bereich der sektorspezifischen Studiengänge (+5) zu beobachten. Vor allem die Bereiche der frühkindlichen Bildung (+2) und der allgemeinen Weiterbildung $(+2)$ wurden ausgeweitet. Zudem kam der Sektor der Bildung im Gesundheits- und Pflegebereich neu hinzu (+3). Der schulspezifische Sektor ist in diesem Zeitraum geschrumpft (-2).

2) Der größte Austausch an Studiengängen entstand im Bereich der sektorenübergreifenden Studiengänge. Fast 40 Prozent der aktuell 23 sektorenübergreifend orientierten Bildungsmanagement-Studiengänge sind jünger als sechs Jahre. Neben den klassischen Themenfeldern werden in diesen jungen Angeboten auch neue Themenzuschnitte erprobt. So rücken beispielsweise Themen der nachhaltigen Entwicklung (ASH Berlin) oder der eEducation (FernUni Hagen) in den Blick.

Zusammenfassend bedeutet dies, dass rein schulspezifische Programme abgenommen haben und sektorenübergreifende Programme durch den Wegfall eines Teils der Programme sowie durch das Hinzukommen neuer Angebote eine neue Durchmischung erfahren haben. Die Beantwortung der Frage, ob diese Verschiebungen innerhalb der Gruppe der sektorenübergreifenden Angebote einen messbaren Effekt 
auf das Durchschnittsprofil eines schulspezifischen oder sektorenübergreifenden Bildungsmanagement-Studiengangs haben, bleibt einer genaueren Analyse der Modulhandbücher vorbehalten. Selbstverständlich ist zu erwarten, dass auch innerhalb der älteren Studienprogramme Aktualisierungen vorgenommen wurden.

\section{Wissensformen des Schulmanagements}

Fragt man sich, was „Schulmanagement“ ist, so kann eine wissenssoziologisch gefasste Antwort darin liegen, Schulmanagement zunächst als einen Sonderwissensbestand zu begreifen (vgl. Berger \& Luckmann, 2013). Bezeichnet ist damit jenes Rollenund Expert ${ }^{\star}$ innenwissen (vgl. ebd., S. $77 \mathrm{ff}$.), das Schulleitungen anwenden, um typische Probleme ihres Handlungsfeldes zu lösen. Dieses anwendungsorientierte Wissen entsteht unter Handlungsdruck und entwickelt sich fort nach Maßgabe seines Problemlösepotenzials (vgl. Krüger, 2016a, S. $74 \mathrm{ff}$.). Entscheidend für die Weiterentwicklung des Wissens ist also, ob neue Wissensbausteine Handlungsprobleme besser lösen können als alte oder eine bessere Lösung zumindest möglich erscheint. Neben diesem Rollenwissen gibt es ein Reflexionswissen über Schulmanagement, das hauptsächlich im wissenschaftlichen Diskurs über Schulmanagement seine Ausdrucksform findet. Hier ist nicht der Erfolg im Handlungsvollzug, sondern seine "höhersymbolische“ Fassung (Dewe, 2014, S. 178) sowie die theoretische und empirische Legitimierbarkeit des Wissens das maßgebliche Kriterium der Weiterentwicklung. Schulmanagement wird hier nicht vollzogen, sondern als empirisch wirksam, modellgeleitet generalisierbar, normativ wünschenswert oder international üblich begründet. Dass zwischen dem Rollen- und dem Reflexionswissen Übersetzungsschwierigkeiten bestehen, wird beispielsweise in den wissenschaftlichen Weiterbildungsstudiengängen spürbar, in denen sich (angehende) Schulleiter ${ }^{\star}$ innen mit einem mitunter praxisfernen Reflexionswissen konfrontiert sehen, obwohl sie sich, zugespitzt formuliert, die Vermittlung von Rezeptwissen (,what works!“) erhofft hatten (vgl. hierzu auch Arnold, 2003, S. 88).

Neben den beiden genannten Wissenskontexten übt auch das Lehr-Lernfeld des Schulmanagements, also die Summe aller Fort- und Weiterbildungsformate, in denen Schulmanagement-Wissen weitervermittelt wird, maßgeblichen Einfluss auf individuelle und gesellschaftliche Deutungsmuster bezüglich professionellem Schulleitungshandeln aus. Auch in diesem Feld entwickelt sich das Wissen auf der Grundlage einer systeminternen Eigenlogik weiter. Im Lehr-Lernfeld ist nicht nur entscheidend, welches Wissen funktioniert und/oder legitimiert ist. Entscheidend ist auch, was in begrenzter Zeit gelehrt und gelernt werden kann und welches Wissen unter gleichbleibenden Bedingungen, also „objektiv“, abgeprüft werden kann. Das Wissen wird durch Lehrende „sedimentiert“ (Berger \& Luckmann, 2013, S. 71), um es in möglichst effizienter Form an möglichst viele Personen weiterzu- 
geben. Es bildet sich eine „eigene Pädagogik“ aus (Pfadenhauer, 2005, S. 12). Was entsteht, ist ein Lernwissen, also ein Wissen, das offiziell gelernt werden soll und das sich mitunter wiederum von den Wissensformen der Praktikerinnen und der Wissenschaftler*innen erheblich unterscheiden kann.

\section{Das Lernwissen von Schulmanagement-Studiengängen}

Nach aktuellem Kenntnisstand (vgl. zum Folgenden auch Krüger, 2016a, S. 434533) lässt sich das in den Modulhandbüchern ausgeführte Lernwissen der Bildungsmanagement-Studiengänge zunächst grob in einen Makro-, einen Meso- und einen Mikrobereich einteilen. Die Mikroebene umfasst Module, die sich mit der Gestaltung des unmittelbaren Lehr-Lernprozesses befassen (z.B. Lernen mit neuen Medien). Auf der Meso-Ebene beziehen sich die Inhalte auf die Gestaltung organisationaler Rahmenbedingungen für Lehr-Lernprozesse. Gemeint sind Module wie Führung, Personalmanagement, Projektmanagement, Wissensmanagement oder Qualitätsmanagement. Das Angebot der Bildungsmanagement-Studiengänge hat in diesem Meso-Bereich seinen Schwerpunkt. Auf der Makro-Ebene werden gesamtgesellschaftliche Zusammenhänge thematisiert, wie beispielsweise Trends des lebenslangen Lernens oder rechtliche Grundlagen, die für alle Bildungsorganisationen eines Sektors identisch sind. Der Hauptanteil des Lernwissens eines BildungsmanagementStudiengangs ist also auf der Meso-Ebene angesiedelt, die sich weiter unterteilen lässt in Module, die das Führen von Menschen durch Menschen (Führung, Moderation, Change Management) in den Blick nehmen, und solche, die das strukturelle Führen (Organisationsmanagement, Prozessmanagement, Controlling) fokussieren. Hier gibt es zwischen den Bildungsangeboten (Stand 2013) signifikante Unterschiede. Es können Leadership-Studiengänge (hoher Anteil an personaler Führung) von Management-Studiengängen (hoher Anteil an struktureller Führung) unterschieden werden. Des Weiteren können forschungsstarke Studiengänge an einem eigenen Profil erkannt werden. Daneben gibt es eine große Gruppe an Allrounder-Studiengängen, die keines der drei genannten Spezialisierungs-Profile aufweisen.

Schulbezogene Bildungsmanagement-Studiengänge weisen im Vergleich zur Gesamtgruppe einen eher geringen Anteil ihres Lernwissens im Bereich der strukturellen Führung aus. „Führung in der Schule, so lässt sich schlussfolgern, wird in den entsprechenden Studiengängen vor allem als personale Führung interpretiert“ (Krüger, 2016a, S. 496). Angesichts der Tatsache, dass Schulleitungen einen Großteil ihrer Zeit mit Strukturierungsaufgaben (z.B. Klassenverteilung, Vertretungsplanung), Qualitätssicherung, Finanzmanagement und Projektmanagement zubringen - der Anteil liegt zwischen 23 und 46 Prozent (Huber, Kruse, Tulowitzki, Schwander \& Heimler, 2017, S. 3; Windlinger \& Hostettler, 2014, S. 52f.) -, kann diese Schwerpunktsetzung durchaus kritisch hinterfragt werden (vgl. Krüger \& Tulowitzki, 2019). 
Inwieweit inhaltliche Anpassungen der Modulhandbücher sowie eine veränderte Zusammensetzung der Untersuchungsgruppe zwischen 2013 und 2019 zu signifikanten Änderungen des Durchschnittsprofils von Bildungsmanagement-Studiengängen geführt haben, soll ein weiterer Untersuchungsschritt zeigen.

Neben den untersuchten Master-Studiengängen werden auf Länderebene teils freiwillige, teils verpflichtende Weiterbildungsangebote für Lehrkräfte, die das Amt der Schulleitung anstreben, gemacht. Die Fortbildungsinhalte gruppieren sich um die Themenschwerpunkte Führung, Personal, Kommunikation, Organisation und Kooperation, Qualitäts- und Schulentwicklung und Recht (vgl. Tulowitzki, Hinzen \& Roller, 2019, S. 159). Es ist also sichtbar, dass Fortbildungen einen Teil des Kanons bedienen, den auch die Schulmanagement-Studiengänge abdecken. In welchem Umfang und mit welchen Schwerpunkten die genannten Inhalte im Rahmen der landesweiten Fortbildungen angeboten werden, kann zusammenfassend noch nicht abgeschätzt werden.

Als Zwischenergebnis lässt sich festhalten, dass das Feld der BildungsmanagementStudiengänge weiterhin wächst. Dieser Trend wurde bereits 2013 festgestellt und gilt ungebrochen. Die Institutionalisierung des Bildungsmanagement-Wissens ist damit weiter vorangeschritten, und das, was wir unter Bildungs- oder Schulmanagement verstehen, wird in zunehmendem Maße durch die „offizielle Lehre“, also das Lernwissen der entsprechenden Studiengänge, mitgeprägt. Auffällig ist auch, dass die Zusammenstellung und Abgrenzung der $\mathrm{zu}$ untersuchenden Studiengänge sich 2019 erheblich schwieriger gestaltete als 2013. Der Blick auf organisationale Rahmenbedingungen des Lehrens und Lernens ist in zunehmendem Maße integraler Bestandteil „traditioneller“ erziehungswissenschaftlicher Studiengänge geworden. Darin kann ein weiteres Indiz dafür gesehen werden, dass die Institutionalisierung des Bildungsmanagement-Wissens weiter voranschreitet. Das sich herauskristallisierende sedimentierte Wissen über professionelles Leitungshandeln in Bildungsorganisationen wirkt als soziales Deutungsmuster auch auf Schulleitungen zurück, die selbst nie einen solchen Studiengang besucht haben. Dieses Lernwissen der Bildungsund Schulmanagementstudiengänge wird seinen Einfluss weiter verstärken, wenn es zu einer verbindlichen Schulleitungsqualifikation kommt.

\section{Literatur und Internetquellen}

Arnold, R. (2003). Theoretische Überlegungen und autobiographische Reflexionen zum Bildungsmanagement. In W. Gieseke (Hrsg.), Institutionelle Innensichten der Weiterbildung (S. 85-97). Bielefeld: Bertelsmann.

Berger, P. L., \& Luckmann, T. (2013). Die gesellschaftliche Konstruktion der Wirklichkeit. Eine Theorie der Wissenssoziologie (25. Aufl.; unveränd. Nachdr. der 5. Aufl.). Frankfurt a. M.: Fischer. 
Dewe, B. (2014). Transformation wissenschaftlicher Informationen in Praxisdeutungen: Über die Wirkung von sozialen und kognitiven Strukturen zur Herstellung von Wissenseffekten in professionellen Beratungszusammenhängen. In U. Unterkofler \& E. Oestreicher (Hrsg.), Theorie-Praxis-Bezüge in professionellen Feldern. Wissensentwicklung und -verwendung als Herausforderung (S. 177-198). Opladen: Budrich. https://doi.org/10.2307/j.ctvddzx9z.12

Huber, S. G., Kruse, C., Tulowitzki, P., Schwander, M., \& Heimler, M. (2017). Arbeitsplatzanalyse Schulleitung Volksschule Kanton Aargau - Executive Summary. Zug: Pädagogische Hochschule, Institut für Bildungsmanagement und Bildungsökonomie.

Krüger, M. (2016a) Das Lernwissen der Bildungsmanagement-Studiengänge im deutschsprachigen Raum. Seine Entstehung sowie seine Formen und Auswirkungen. Hamburg: Dr. Kovač.

Krüger, M. (2016b). Bildungsmanagement-Studiengänge und die Formen ihres Einflusses auf Deutungsmuster professionellen Handelns. ZfW - Zeitschrift für Weiterbildungsforschung, 39 (3), 297-315. https://doi.org/10.1007/s40955-016-0075-8

Krüger M., \& Tulowitzki, P. (2019). La formation professionnelle des responsables détablissement scolaire en Suisse alémanique et son influence sur la perception du métier. In L. Progin, E. Richard, G. Pelletier \& E. Richard (Hrsg.), Diriger un établissement scolaire - Tensions, ressources et développement (S. 143-158). Brüssel: De Boeck.

Pfadenhauer, M. (2005). Die Definition des Problems aus der Verwaltung der Lösung. In M. Pfadenhauer (Hrsg.), Professionelles Handeln (S. 9-22). Wiesbaden: VS.

Tulowitzki, P., Hinzen, I., \& Roller, M. (2019). Die Qualifizierung von Schulleiter*innen in Deutschland - ein bundesweiter Überblick. DDS - Die Deutsche Schule, 111 (2), 149170. https://doi.org/10.31244/dds.2019.02.04

Windlinger, R., \& Hostettler, U. (2014). Schulleitungshandeln im Kontext. Zum Stand der geleiteten Schulen im Kanton Bern aus der Perspektive der Schulleitenden, der Lehrpersonen und der Kollegien (Beiträge für die Praxis, Bd. 2). Bern: hep.

Michael Krüger, Dr., wissenschaftlicher Mitarbeiter am Institut für Bildungsmanagement der Pädagogischen Hochschule Ludwigsburg.

E-Mail: krueger@ph-ludwigsburg.de

Korrespondenzadresse: Pädagogische Hochschule Ludwigsburg, Institut für Bildungsmanagement, Reuteallee 46, 71634 Ludwigsburg 